\title{
Magnetic properties of ${ }^{177} \mathrm{Hf}$ and ${ }^{180} \mathrm{Hf}$ in the strong-coupling deformed model
}

\author{
S. Muto,,${ }^{1, *}$ N. J. Stone, ${ }^{2,3}$ C. R. Bingham,,${ }^{2,4}$ J. R. Stone, ${ }^{2,3}$ P. M. Walker, ${ }^{5}$ G. Audi, ${ }^{6}$ C. Gaulard, ${ }^{6}$ U. Köster ${ }^{7}$ J. Nikolov, ${ }^{8}$ \\ K. Nishimura, ${ }^{9}$ T. Ohtsubo, ${ }^{10}$ Z. Podolyak, ${ }^{5}$ L. Risegari, ${ }^{6}$ G. S. Simpson,,${ }^{11}$ M. Veskovic,${ }^{8}$ and W. B. Walters ${ }^{12}$ \\ ${ }^{1}$ Neutron Science Laboratory, KEK, Tsukuba, Ibaraki 305-0801, Japan \\ ${ }^{2}$ Department of Physics and Astronomy, University of Tennessee, Knoxville, Tennessee 37996, USA \\ ${ }^{3}$ Department of Physics, University of Oxford, Oxford OX1 3PU, United Kingdom \\ ${ }^{4}$ Oak Ridge National Laboratory, Oak Ridge, Tennessee 37831, USA \\ ${ }^{5}$ Department of Physics, University of Surrey, Guildford, Surrey GU2 7XH, United Kingdom \\ ${ }^{6}$ CSNSM, F-91405 Orsay, France \\ ${ }^{7}$ Institut Laue Langevin, F-38042 Grenoble, France \\ ${ }^{8}$ Department of Physics, University of Novi Sad, 21000 Novi Sad, Serbia \\ ${ }^{9}$ Faculty of Engineering, Toyama University, Toyama 930, Japan \\ ${ }^{10}$ Department of Physics, Niigata University, Niigata 950-2181, Japan \\ ${ }^{11}$ LPSC, F-38026 Grenoble, France \\ ${ }^{12}$ Department of Chemistry and Biochemistry, University of Maryland, College Park, Maryland 20740, USA
}

(Received 14 January 2014; published 7 April 2014)

\begin{abstract}
This paper reports NMR measurements of the magnetic dipole moments of two high- $K$ isomers, the $37 / 2^{-}$, $51.4 \mathrm{~m}, 2740 \mathrm{keV}$ state in ${ }^{177} \mathrm{Hf}$ and the $8^{-}, 5.5 \mathrm{~h}, 1142 \mathrm{keV}$ state in ${ }^{180} \mathrm{Hf}$ by the method of on-line nuclear orientation. Also included are results on the angular distributions of $\gamma$ transitions in the decay of the ${ }^{177} \mathrm{Hf}$ isotope. These yield high precision $E 2 / M 1$ multipole mixing ratios for transitions in bands built on the $23 / 2^{+}, 1.1 \mathrm{~s}$, isomer at $1315 \mathrm{keV}$ and on the $9 / 2^{+}, 0.663 \mathrm{~ns}$, isomer at $321 \mathrm{keV}$. The new results are discussed in the light of the recently reported finding of systematic dependence of the behavior of the $g_{R}$ parameter upon the quasiproton and quasineutron make up of high- $K$ isomeric states in this region.
\end{abstract}

DOI: 10.1103/PhysRevC.89.044309

PACS number(s): 21.10.Ky, 21.60.Cs, 27.70.+q, 29.30.Lw

\section{INTRODUCTION}

This paper reports measurement of the magnetic dipole moments of the $51.4 \mathrm{~m}, 2740 \mathrm{keV}, 37 / 2^{-}$isomer in ${ }^{177} \mathrm{Hf}$ and of the $5.5 \mathrm{~h}, 1142 \mathrm{keV}, 8^{-}$isomer in ${ }^{180} \mathrm{Hf}$, using the method of NMR on oriented nuclei, at the on-line NICOLE facility, ISOLDE, CERN. In addition, measurements of the angular distribution of $\gamma$ transitions from the ${ }^{177} \mathrm{Hf}^{\mathrm{m} 2}$ isomer are analyzed to give precise values of the $E 2 / M 1$ mixing ratios in transitions in bands built upon the $23 / 2^{+}, 1.1 \mathrm{~s}, 1315 \mathrm{keV}$ isomer and the $9 / 2^{+}, 321 \mathrm{keV}$ state of this nucleus.

Accurate measurements of the electromagnetic properties of ground states, isomers, and rotational band states in deformed nuclei yield parameters of the deformed nuclear model used to describe these nuclei. In the region between $\mathrm{Yb}$ and $\mathrm{W}$ ground states and isomers have close-to-constant deformation and the parameters of the model can be explored thoroughly. In this work the main focus is on isomeric state, band-head, magnetic dipole moments to yield values of the single particle $g$ factor, $g_{K}$, with good precision. In addition, analysis of the angular distribution of mixed $M 1 / E 2$ transitions in the decay of the isomers studied yields values of the mixing ratio $\delta$ which is related to the $g$ factors $g_{K}$, and $g_{R}$, and intrinsic quadrupole moment $Q_{0}$, of the band head [see Eq. (4)].

Interest in obtaining the magnetic parameters $g_{K}$ and $g_{R}$ in these states derives from their involvement with ideas of superconductivity in nuclei. In the strong coupling model of

${ }^{*}$ Deceased. well-deformed nuclei the influence of the quasiparticle state upon the band properties arises through the dependence of the pairing strength, and hence the pairing gap $\Delta$, upon the available orbitals close to the Fermi surface. In the deformed potential each state is doubly degenerate and is available for pair scattering if empty, however the occupation of a state by a single quasiparticle renders it unavailable to pair scattering. This is the process of blocking [1,2]. Since the pairing gap is determined by the number of pairs and the numbers of states between which they can scatter, increasing the number of quasiparticles increases blocking and reduces the gap $\Delta$.

These considerations apply separately to protons and neutrons. Where the rotational energy levels of the bands are concerned, the relevant model parameter is the total moment of inertia $\mathcal{I}_{\text {tot }}=\mathcal{I}_{p}+\mathcal{I}_{n}$. The stronger the pairing associated with the state the more $\mathcal{I}_{\text {tot }}$ is reduced compared to the classical rigid body value. Blocking of either proton or neutron orbitals weakens the pairing and increases $\mathcal{I}_{\text {tot }}$. When electromagnetic properties of the band are considered, transition matrix elements, intensity ratios, multipole mixing ratios and the collective $g$ factor $g_{R}$ show different sensitivity to proton and neutron pairing, and the effects of blocking, through their differing effective charges, crudely 1 for protons and 0 for neutrons. In this limit $g_{R}$ is given by the ratio $\mathcal{I}_{p} / \mathcal{I}_{\text {tot }}$, which yields the familiar simple result $g_{R} \sim Z / A$ for rigid rotation of the whole nucleus. It follows from these basic ideas that we can expect any increase in, for example, neutron blocking, by reducing the neutron pairing gap and increasing the neutron contribution to $\mathcal{I}_{\text {tot }}$, to reduce $g_{R}$ relative to $Z / A$, while increased proton blocking will have the opposite result. 
The new experimental results presented here, in combination with a much larger body of data from existing literature, have been used recently to explore these concepts [3]. It has been shown that, within the rather limited data presently available, the principle of additivity can be relied upon to give good estimates of the single particle $g$ factor, $g_{K}$, in multi-quasi-particle isomers. The use of this principle to estimate $g_{K}$ in many bands revealed a wide, systematic, dependence of the collective $g$ factor, $g_{R}$, upon the neutron and proton quasiparticle make-up of the band-head states, presenting a new challenge to the theory of superconductivity, pairing and blocking in these deformed nuclei. This finding gives additional reason to extend, in particular, band-head magnetic moment measurements of good accuracy and to seek accurate mixing ratios to yield $g_{R}$, as are reported here.

The theoretical context of this work is given in Sec. II. Experimental details and new data on the angular properties of gamma transitions in the decay of the $37 / 2^{-} K$ isomer of ${ }^{177} \mathrm{Hf}$ are described in Secs. III and IV together with NMR results which yield the magnetic dipole moments of the $37 / 2^{-}$ $K$ isomer and of the $8^{-}$isomer in ${ }^{180} \mathrm{Hf}$. Consistency of the new results with the findings of [3] is discussed in Sec. V.

\section{THEORETICAL FRAMEWORK}

Relevant expressions from the strong coupling model concerning electromagnetic properties of the nuclear states and transitions are given here [4]. For the static magnetic dipole moment of a band state of spin $I$ based on a band head of $\operatorname{spin} K$

$$
\mu=g_{R} I+\left(g_{K}-g_{R}\right) \frac{K^{2}}{I+1}
$$

which, for the band head $K=I$, gives

$$
\mu=g_{K}\left[I^{2} /(I+1)\right]+g_{R}[I /(I+1)] .
$$

Since the values of spin $I$ in this paper are large, this expression makes it clear that the moment has only weak dependence upon the "collective $\mathrm{g}_{R}$ factor and is largely determined by the quasiparticle $g_{K}$ factor.

For a pure multi-quasi-particle state for which $K$ is the simple sum of the constituent quasiparticle $K_{i}$ 's, the quasiparticle $g$ factor $g_{K}$ is given in terms of the constituent quasiparticle $g_{K i}$ factors by

$$
g_{\mathrm{K}}=\frac{1}{K} \sum_{i} K_{i} g_{K_{i}} .
$$

Two spectroscopic variables, the $E 2 / M 1$ ratio $\delta$ in transitions from a level of spin $I$ to a lower level of spin $I-1$ and the branching ratio of transitions from a state of spin $I$ to lower states of spin $I-1$ and $I-2$, depend upon the difference $\left(g_{K}-g_{R}\right)$ of the two (collective and quasiparticle) $g$ factors. These variables can both be written in terms of $\delta$ given by

$$
\delta=\frac{0.933 E_{\gamma} Q_{0}}{\left(g_{K}-g_{R}\right) \sqrt{\left(I^{2}-1\right)}},
$$

where $E_{\gamma}$ is the transition energy in $\mathrm{MeV}$ and $Q_{0}$ is the intrinsic quadrupole moment in $e$ b. From these expressions it is seen that to separate the quasiparticle $g$ factor $g_{K}$ from the rotation $g$ factor $g_{R}$ it is necessary to have data on either transition branching ratios from states in the band to lower states of spin reduced by one and two units or $M 1 / E 2$ mixing ratios for transitions between states of spin differing by one, combined with the static magnetic dipole moment of a state of the band. Of these, the static dipole moments are the rarest. In addition, knowledge of the intrinsic quadrupole moment $Q_{0}$ of the band states is required.

\section{EXPERIMENTAL DETAILS}

\section{A. Nuclear orientation of the $37 / 2^{-}, 51.4 \mathrm{~m}, K$ isomer in ${ }^{177} \mathrm{Hf}$}

At the ISOLDE isotope separator facility, CERN, new results have been obtained on the magnetic moment of the $37 / 2^{-}$high-spin $K$ isomer and on $\gamma$ transitions in its decay to the ground state by the method of on-line nuclear orientation combined with NMR [5]. The $1.4 \mathrm{GeV}$ proton beam from CERN's PSB synchrotrons was incident upon a mixed tantalum/tungsten foil target and separation of the $\mathrm{Hf}$ isotopes was achieved by introducing $\mathrm{CF}_{4}$ gas into the plasma ion source and extracting a beam of $\mathrm{HfF}_{3}^{+}$ions [6]. The ions, accelerated through $60 \mathrm{keV}$, impinged upon a pure iron foil soldered to the cold finger of the NICOLE on-line nuclear orientation system [7] maintained at temperatures down to about $12 \mathrm{mK}$. The $\mathrm{HfF}_{3}^{+}$ions disintegrated at the foil surface and the Hf activity was implanted into the Fe lattice. By interrupting the implantation and allowing the dilution refrigerator to cool further, data were taken to about $6 \mathrm{mK}$ as the activity decayed. The iron foil was magnetized by an applied magnetic field of $0.5 \mathrm{~T}$.

At mass $A=177$ the $\mathrm{Hf}$ beam contains both the isomer ${ }^{177} \mathrm{Hf}^{\mathrm{m} 2}\left(I^{\pi}=37 / 2^{-} T_{1 / 2}=51.4 \mathrm{~m}\right)$ and stable ${ }^{177} \mathrm{Hf}$ ground state nuclei. The isomer activity made up about $1 \%$ of the beam. The decay scheme of ${ }^{177} \mathrm{Hf}^{\mathrm{m} 2}$ is shown in Fig 1. Angular distribution parameters for all transitions were obtained from measurements, as a function of the foil temperature, of the $\gamma$ photopeak count rates in two pairs of high resolution Ge detectors placed at angles of 0, 90, 90, and 180 degrees to the axis of polarization. The counts were normalized to rates measured with the activity unoriented at a temperature close to $1 \mathrm{~K}$. The foil temperature was obtained from measurements of the angular distributions of the $1333 \mathrm{keV}$ and $1173 \mathrm{keV}$ transitions in the decay of ${ }^{60} \mathrm{Co}$ activity in a Co single crystal soldered to the back of the cold finger. Since, for the ${ }^{60} \mathrm{CoCo}$ system, all orientation parameters are known, these measurements act as an accurate thermometer in the region between about $50 \mathrm{mK}$ and $5 \mathrm{mK}$.

High degrees of polarization were achieved, produced by the large hyperfine field experienced by the Hf activity in the iron lattice. Examples of cold, oriented, gamma spectra compared with warm $\left(1 \mathrm{~K}\right.$, unoriented) spectra in $0^{\circ}$ and $90^{\circ}$ detectors are shown in Fig. 2, in which the strongly differing anisotropies in transitions between levels in different bands of the decay are clearly seen.

In addition to $\gamma$ transition angular distribution measurements, NMR of the implanted ${ }^{177} \mathrm{Hf}^{\mathrm{m} 2}$ parent state activity was sought by exposing the implanted nuclei to an RF field, 


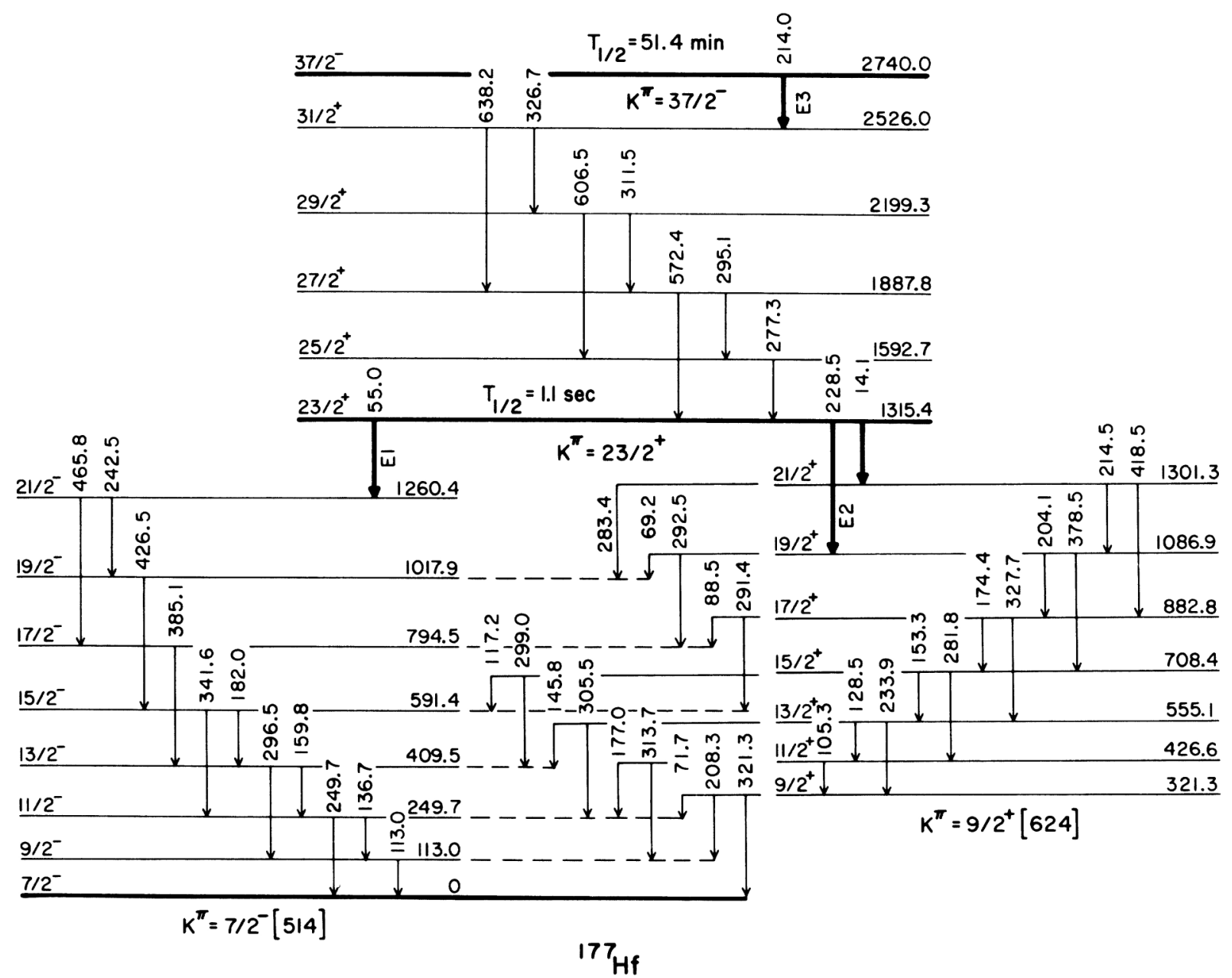

FIG. 1. ${ }^{177} \mathrm{Hf}^{\mathrm{m} 2}$ decay scheme. Taken from [19].

transverse to the axis of polarization, produced by a simple two-turn coil.
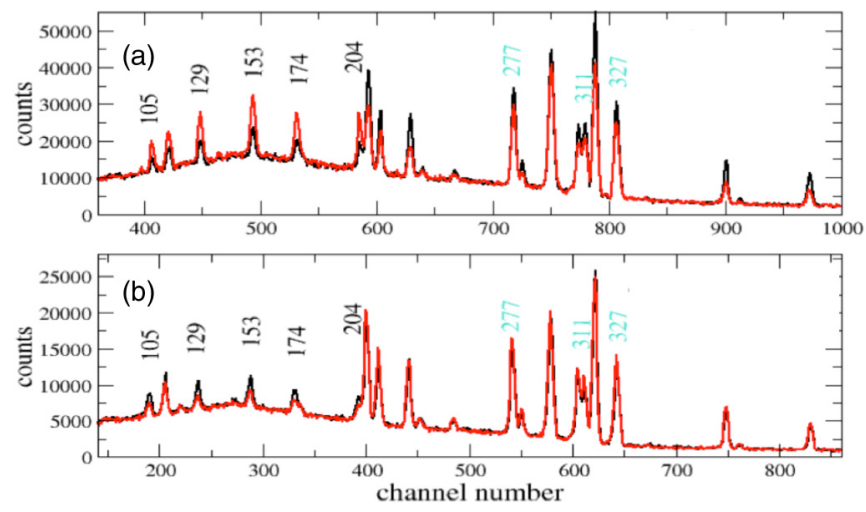

FIG. 2. (Color online) Warm and cold spectra from $0^{\circ}$ and $90^{\circ}$ detectors for two series of $E 2 / M 1$ transitions. Black/dark energy labels-in band built on $9 / 2^{+}$[624] state-note increases at $0^{\circ}$. Blue/pale energy labels -in band built on $23 / 2^{+}$three-quasiparticle isomer-note decreases at $0^{\circ}$.

\section{B. Hyperfine interaction and nuclear spin-lattice relaxation time estimates}

Implanted nuclei have a high probability of coming to rest in regular lattice sites and the well-defined hyperfine interaction allows observation of nuclear magnetic resonance by the disturbance caused to the gamma decay angular distribution by resonant absorption of RF energy. However even though the foil lattice may be at millikelvin temperature the nuclei are implanted unoriented and it takes a time, related to the nuclear spin-lattice relaxation time, $T_{1}$ for nuclear polarization to be established [8]. Both the resonant NMR frequency, $v_{\text {res }}$ and $T_{1}$ depend upon the strength of the hyperfine structure splitting of the state undergoing resonance and/or orientation, $h v_{\mathrm{res}}=\mu B_{\mathrm{hf}} / I$.

The nuclear moment of the $37 / 2^{-}$isomer can be estimated to be $\sim 7 \mu_{N}$ (see Sec. IVE below) and the hyperfine field at $\mathrm{Hf}$ in $\mathrm{Fe}$ is $B_{\mathrm{hf}}=-67.4(9) \mathrm{T}$ ([9] improved using ${ }^{175} \mathrm{Hf}$ moment $0.677(9)$ [10]). These values yield $v_{\text {res }}$ in the region of $200 \mathrm{MHz}$. There have been no spin lattice relaxation time measurements for $\mathrm{Hf}$ isotopes in iron, however an empirical relationship, $C_{K} T_{\text {int }}^{2}=1.4 \times 10^{-4} \mathrm{sK}^{3}$, where $\mathrm{T}_{\text {int }}$ is the nuclear interaction strength, $T_{\mathrm{int}}=\mu B_{\mathrm{hf}} / k I$, can be used to give estimates of the Korringa constant $C_{K}$, usually 
found reliable to better than a factor of two [8]. The resulting prediction is $T_{1}\left(37 / 2^{-}\right) \sim 19 \mathrm{~s}$ at $10 \mathrm{mK}$ which is much less than the isomer lifetime of $51.4 \mathrm{~m}$, so full thermal equilibrium between the ${ }^{177} \mathrm{Hf}^{\mathrm{m} 2}$ nuclei and the lattice will be established prior to decay.

There remains the possibility that orientation may be perturbed by further interaction with the lattice during the lifetime of the $1.1 \mathrm{~s} 23 / 2^{+}$isomeric state at $1315 \mathrm{keV}$. Any such re-orientation would affect observed anisotropies in transitions below the isomer. However, an estimate of $T_{1}$ for the $23 / 2^{+}$ isomer, based on an estimated $23 / 2^{+}$moment and the above $T_{1}$ $\left(37 / 2^{-}\right)$estimate gives the value $T_{1}\left(25 / 2^{+}\right) \sim 8 \mathrm{~s}$ at $10 \mathrm{mK}$. This is substantially longer than the lifetime of the isomer, indicating that any reorientation effect will be small.

\section{Nuclear orientation of the $8^{-}, 5.5 \mathrm{~h}, 1142 \mathrm{keV}$, state in ${ }^{180} \mathrm{Hf}$}

At mass 180 the $\mathrm{Hf}$ beam has a strong isomeric ${ }^{180} \mathrm{Hf}$ component which decays by mixed $E 1 / M 2 / E 3$ transitions to $8^{+}$and $6^{+}$states in the ground state band. These states decay by a sequence of pure E2 transitions which show strong anisotropy as has been reported previously [11]. Earlier measurements of the magnetic dipole moment of this isomer have not been of high accuracy. Körner et al. [12] reported 8.7(10) $\mu_{N}$ by a Mössbauer method and Krane et al. [13] 9.0(9) $\mu_{N}$ by an integral nuclear orientation experiment on sources of ${ }^{180} \mathrm{Hf}^{\mathrm{m}}$ in $\mathrm{HfZrFe}_{2}$ alloys. Since NMR on the implanted activity would provide a more precise result for the moment, resonance was sought with the activity implanted into an Fe foil. Unfortunately, at the high frequency at which resonance is expected for this isomer (in the region of $520 \mathrm{MHz}$ ) the RF line into the Nicole dilution refrigerator shows strong standing wave resonances which result in non-nuclear-resonance heating of the sample and render true resonance unobservable. In a second experiment the activity was implanted into a pure $\mathrm{Ni}$ foil in which the magnetic hyperfine field at Hf nuclei is much reduced. Resonance was observed at a frequency of 92.2(2) MHz in an applied field of $0.10 \mathrm{~T}$. The result is discussed further below.

\section{ANALYSIS OF RESULTS}

\section{A. $\gamma$ transition anisotropies}

Photopeak counts for each transition in each spectrum for each $\gamma$ detector were obtained using the fitting code DAMM. Counts taken with the sample at temperatures with appreciable orientation were normalized by taking ratios with counts from unoriented, warm (1 K), spectra. The gamma transition anisotropies, $A$, were obtained for each of the two pairs of detectors using the expressions

$$
W(\theta, T)=N(\theta, T) / N(\theta, \text { warm })
$$

and

$$
A=\left[W\left(0^{0}, T\right) / W\left(90^{0}, T\right)-1\right] .
$$

The anisotropies were analysed further using the standard nuclear orientation formalism [14]

$$
W(\theta, T)=1+f \sum_{\lambda} B_{\lambda} U_{\lambda} A_{\lambda} Q_{\lambda} P_{\lambda}(\cos \theta) .
$$

Here $f$ is the fraction in good sites. The $B_{\lambda}$ factors describe orientation of the parent, isomeric state and depend upon the strength of the hyperfine interaction, the spin and the temperature. The $U_{\lambda}$ factors are calculated for each state below the parent and require knowledge of the spins of any intervening states, the intensities of all transitions between them and their multipolarity, with admixtures treated without regard to phase. The $A_{\lambda}$ parameters describe the observed emission and depend upon the initial and final state spins and the multipolarity, treated with sensitivity to phase of any multipole mixing ratio $\delta . Q_{\lambda}$ are correction factors which account for the finite solid angle subtended by the detectors and $P_{\lambda}$ are the associated Legendre polynomials. Since $\gamma$ emission conserves parity, the summation has only even terms (up to $\lambda=2 L_{\max }$, where $L_{\max }$ is the highest multipolarity in the observed emission).

In all on-line nuclear orientation experiments it has been found that the angular distributions can be well described as consisting of two components, the first from nuclei coming to rest in undisturbed (good) lattice sites and subject to the full hyperfine interaction and the second unoriented at all temperatures. The unoriented fraction describes nuclei which are stopped in the thin oxide layer which is always present on the implantation foil surface or are stopped in other very disturbed regions which are not magnetically ordered.

\section{B. Analysis of the pure $E 2, \Delta I=2$, transitions: Determination of the fraction in good sites}

The first transition emitted by the $37 / 2^{-}$isomer is the $214 \mathrm{keV}$ pure E3 transition. However this is an unresolved doublet with a mixed transition lower in the decay scheme so could not be used to evaluate the fraction $f$. The $U_{\lambda}$ values for this pure multipole transition are known, however, so $f$ can be obtained from the anisotropy of the pure $E 2,638 \mathrm{keV}$, $\left(31 / 2^{+}-27 / 2^{+}\right)$transition. As the other parameters necessary to calculate $U_{\lambda}$ for lower states can all be extracted from the experimental data, values for $f$ can also be found from data on the other $E 2$ transitions between pairs of lower states built on the $23 / 2^{+} K$ isomer. The results are given in Table I. As an example, in Fig. 3 are shown the data for $606.5 \mathrm{keV}$ transition

TABLE I. Fraction $f$ of implanted Hf nuclei in good sites as extracted from fitting $E 2$ transitions with known decay parameters.

\begin{tabular}{lccl}
\hline \hline \multicolumn{2}{c}{ Transition } & Energy & Fitted fraction $f$ \\
\cline { 1 - 2 }$I_{1}$ & $I_{2}$ & $(\mathrm{keV})$ & \\
& \multicolumn{4}{c}{ Above the $23 / 2^{+}$isomer } \\
$31 / 2$ & $27 / 2$ & 638.2 & $0.755(7)$ \\
$29 / 2$ & $25 / 2$ & 606.5 & $0.786(12)$ \\
$27 / 2$ & $23 / 2$ & 572.4 & $0.768(12)$ \\
\multicolumn{4}{c}{ Below the $23 / 2^{+}$isomer } \\
$23 / 2$ & $19 / 2$ & 228.5 & $0.773(6)$ \\
$21 / 2$ & $17 / 2$ & 418.5 & $0.773(13)$ \\
$19 / 2$ & $15 / 2$ & 378.5 & $0.773(6)$ \\
$15 / 2$ & $11 / 2$ & 281.8 & $0.779(9)$ \\
$13 / 2$ & $9 / 2$ & 233.9 & $0.802(21)$ \\
\hline \hline
\end{tabular}




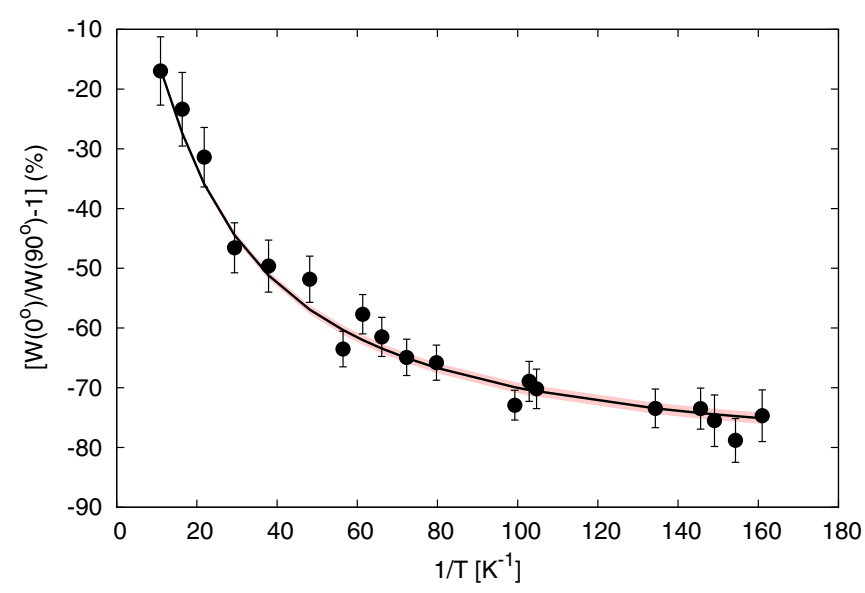

FIG. 3. (Color online) Anisotropy of the $606.5 \mathrm{keV} \mathrm{29/2^{+ } -}$ $25 / 2^{+}$pure $E 2$ transition in band built on $23 / 2^{+}$isomer. Fitted curve with shaded band is for $f=0.786(12)$.

between the $29 / 2^{+}$and $25 / 2^{+}$states and calculation using the fit $f$ value, with its uncertainty.

\section{Reorientation in the $23 / 2^{+}$isomeric state}

This state has lifetime $1.1 \mathrm{~s}$ and, although estimates given above suggest that the spin-lattice relaxation time $T_{1}$ of this state is considerably longer than the lifetime, this point requires further attention if anisotropies of $\gamma$ transitions between states below the isomer are to be quantitatively analyzed. Data on anisotropies of the series of pure $E 2$ transitions between states below the $23 / 2^{+}$isomer have all be treated to yield values of $f$ on the assumption that reorientation may be neglected. The results are given in Table I. The close agreement between values of $f$ obtained from transitions above and below the isomer show clearly that the assumption is indeed valid. In Fig. 4 the data on the $228.5 \mathrm{keV}$ transition between the $23 / 2^{+}$

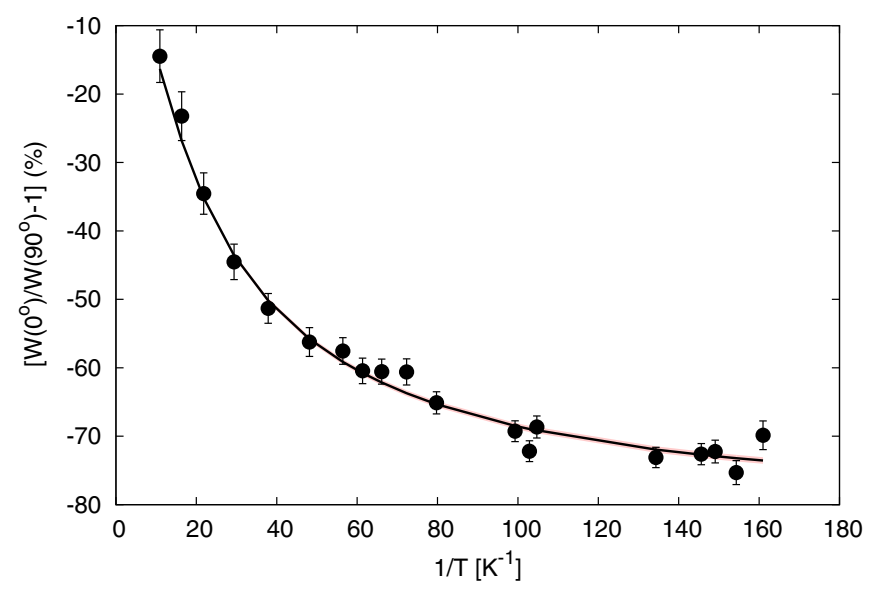

FIG. 4. (Color online) Anisotropy of the $228.5 \mathrm{keV} 23 / 2^{+}-$ $19 / 2^{+}$pure $E 2$ transition from the $23 / 2^{+}$isomer to the band built on the $9 / 2^{+}$[624] band. Fitted curve with shaded band is for $f=$ $0.773(6)$.
TABLE II. E2/M1 mixing ratios in transitions from levels of ${ }^{177} \mathrm{Hf}$.

\begin{tabular}{|c|c|c|c|c|c|c|}
\hline$K$ & $\begin{array}{l}\text { Level } \\
\text { spin }\end{array}$ & $\begin{array}{c}E_{\gamma} \\
(\mathrm{keV})\end{array}$ & $\begin{array}{c}\delta(\text { In beam }) \\
{[15]}\end{array}$ & $\begin{array}{c}\delta\left({ }^{177} \mathrm{Lu}\right) \\
{[16]}\end{array}$ & $\begin{array}{c}\delta\left({ }^{177} \mathrm{Hf}\right) \\
\text { this work }\end{array}$ & $\left(g_{K}-g_{R}\right) / Q_{0}$ \\
\hline \multirow[t]{2}{*}{$37 / 2$} & $41 / 2$ & 420.9 & $0.74(23)$ & & & $0.025(8)$ \\
\hline & $43 / 2$ & 440.0 & $0.74(16)$ & & & $0.025(5)$ \\
\hline \multirow[t]{8}{*}{$23 / 2$} & $25 / 2$ & 277.3 & & & $0.302(4)$ & $0.069(1)$ \\
\hline & $27 / 2$ & 295.1 & $0.31(2)$ & & & \\
\hline & $29 / 2$ & 311.5 & $0.36(2)$ & & $0.285(5)$ & $0.071(2)$ \\
\hline & $31 / 2$ & 326.5 & $0.30(1)$ & & $0.278(5)$ & $0.071(2)$ \\
\hline & $33 / 2$ & 340.1 & $0.26(1)$ & & & \\
\hline & $35 / 2$ & 351.9 & $0.21(1)$ & & & \\
\hline & $37 / 2$ & 361.7 & $0.18(2)$ & & & \\
\hline & $39 / 2$ & 369.4 & $0.20(2)$ & & & \\
\hline \multirow[t]{10}{*}{$9 / 2$} & $11 / 2$ & 105.3 & & $-0.36(4)$ & $-0.23(4)$ & $-0.083(15)$ \\
\hline & $13 / 2$ & 128.4 & $0.38(1)$ & $-0.37(6)$ & $-0.34(3)$ & $-0.055(5)$ \\
\hline & $15 / 2$ & 153.1 & $0.38(1)$ & $-0.33(5)$ & $-0.317(13)$ & $-0.061(3)$ \\
\hline & $17 / 2$ & 174.3 & $0.36(1)$ & $-0.32(4)$ & $-0.296(13)$ & $-0.065(3)$ \\
\hline & $19 / 2$ & 204.1 & $0.40(1)$ & $-0.33(5)$ & $-0.289(13)$ & $-0.070(4)$ \\
\hline & $21 / 2$ & 214.3 & $0.35(1)$ & $-0.29(2)$ & & \\
\hline & $23 / 2$ & 260.5 & $0.50(2)$ & & & \\
\hline & $25 / 2$ & 241.8 & $0.40(2)$ & & & \\
\hline & $27 / 2$ & 325.4 & $0.61(7)$ & & & \\
\hline & $29 / 2$ & 249.4 & $0.27(2)$ & & & \\
\hline
\end{tabular}

and $19 / 2^{+}$states are shown, with calculation using the fit $f$ value, with its uncertainty.

The data from both pairs of detectors were in very close agreement. An average value $f=0.775(5)$ was adopted and used throughout the analysis.

\section{Analysis of the mixed multipole $\Delta I=1$ transitions: Determination of the $E 2 / M 1$ mixing ratios}

Each state in the bands built upon the $23 / 2^{+}$multi-quasiparticle isomer and the $9 / 2^{+}$and $7 / 2^{-}$single quasiparticle states decays both by a direct $E 2, \Delta I=2$, transition and a mixed $E 2 / M 1, \Delta I=1$, transition. The $E 2 / M 1$ multipole

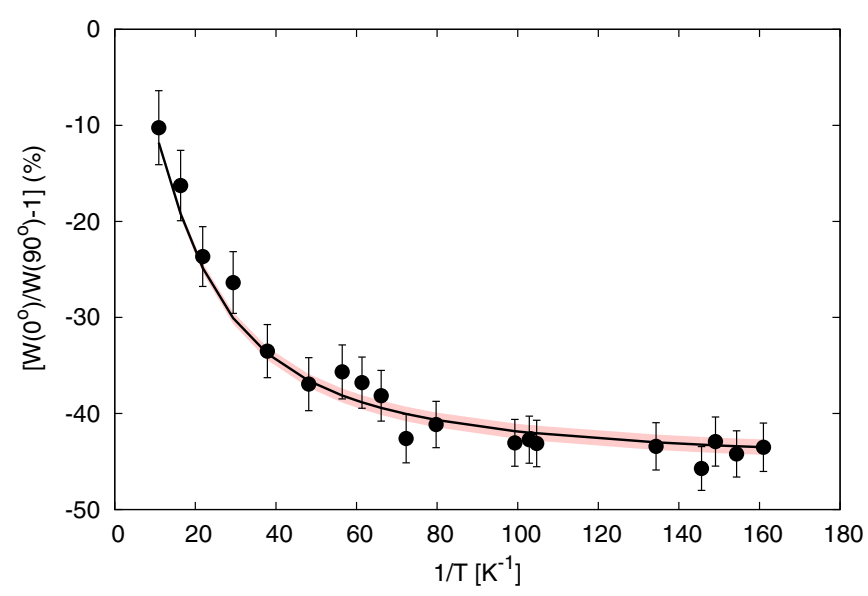

FIG. 5. (Color online) Anisotropy of the $277.3 \mathrm{keV} 25 / 2^{+}-$ $23 / 2^{+}$mixed $E 2 / M 1$ transition in band built on $23 / 2^{+}$isomer. Fitted curve with shaded band is for $\delta[E 2 / M 1]=+0.302(4)$. 


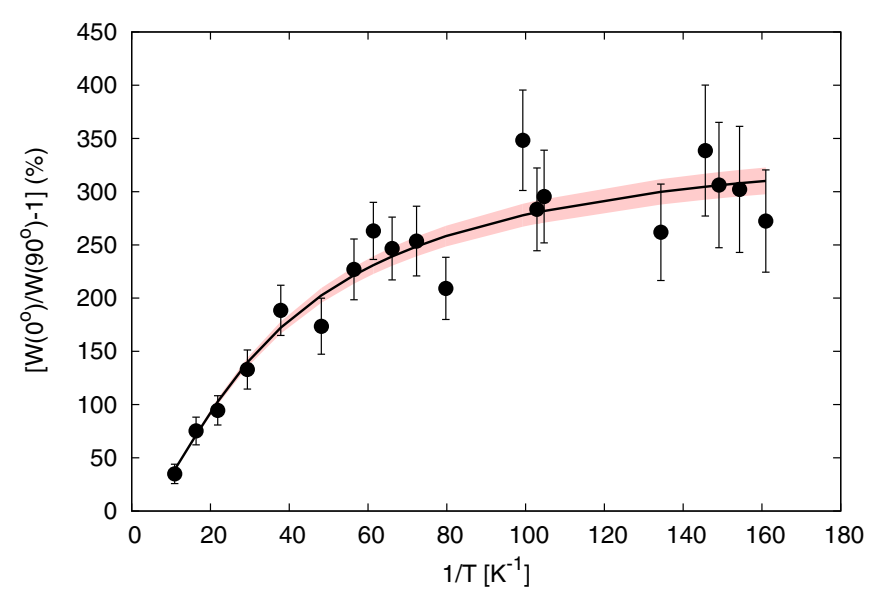

FIG. 6. (Color online) Anisotropy of the $153.1 \mathrm{keV} 15 / 2^{+}-$ $13 / 2^{+}$mixed $E 2 / M 1$ transition in band built on $9 / 2^{+}[624]$ band. Fitted curve with shaded band is for $\delta[E 2 / M 1]=-0.317(13)$.

mixing ratio, $\delta$, is a most useful quantity in establishing the parameters of the band, as described previously. For each of these transitions the anisotropies were analysed in a somewhat novel way to give the best fit values of $\delta$. Each data point in the temperature dependence (19 in all) was regarded as an individual experiment and fitted with $\delta$ as the only unknown. The resulting set of $\delta$ s was treated statistically to give the best fit value and the error on this value. Results are shown in Table II, compared to results from other investigations $[15,16]$. The overall agreement is excellent and the new results have significantly smaller experimental uncertainties. Examples of the fits are shown in Figs. 5 and 6. Figure 5 shows data for the $277.3 \mathrm{keV}$ transition between the $25 / 2^{+}$and $23 / 2^{+}$states in the $23 / 2^{+}$band. All mixed transitions in this band show similar negative anisotropies. Figure 6 shows data for the $153.1 \mathrm{keV}$ transition between the $15 / 2^{+}$and $13 / 2^{+}$states in the $9 / 2^{+}$ band. All mixed transitions in this band show similar large positive anisotropies. The larger scatter and uncertainties for these transitions arise because they are measured on a large Compton-backscattered $\gamma$ intensity so that the small $90^{\circ}$ counts at lower temperatures have large uncertainties (see Fig. 2).

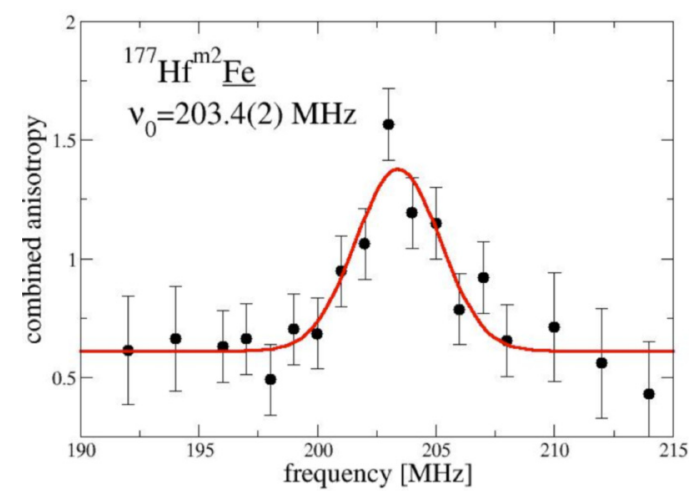

FIG. 7. (Color online) Resonance observed in a combined signal from the stretched $E 2$ transitions in the decay of the $37 / 2^{-}$isomer in ${ }^{177} \mathrm{Hf}$.

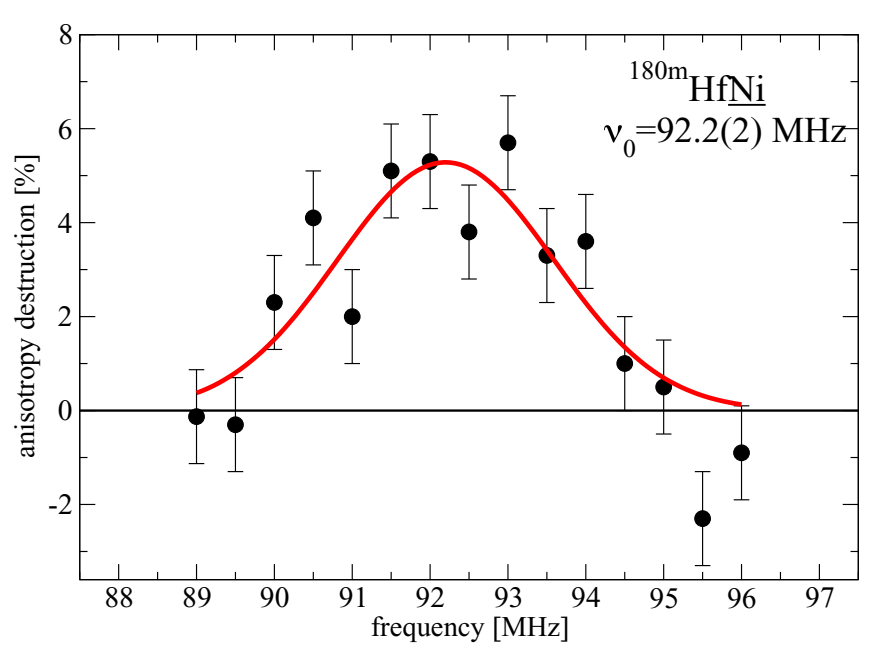

FIG. 8. (Color online) Resonance observed in percentage destruction of anisotropy of the $8^{-}$isomer in ${ }^{180} \mathrm{Hf}$.

\section{E. The magnetic moment of the $37 / 2^{-}$isomer in ${ }^{177} \mathrm{Hf}$}

The search for NMR of the $37 / 2^{-}$isomer was carried out with RF swept between 180 and $230 \mathrm{MHz}$ in $1 \mathrm{MHz}$ steps with RF modulation of $1 \mathrm{MHz}$. A positive signal was found, as shown in Fig. 7, with center frequency

$$
v_{\text {res }}\left({ }^{177} \mathrm{Hf}^{\mathrm{m} 2} \underline{\mathrm{Fe}}\right)=203.4(2) \mathrm{MHz} .
$$

This frequency, combined with the known hyperfine field $-67.4(9) \mathrm{T}$, adjusted for the external field of $0.100 \mathrm{~T}$ applied to the Fe foil during the NMR measurements, yields the magnitude of the magnetic moment of the $37 / 2^{-}$isomer as

$$
|\mu|\left({ }^{177} \mathrm{Hf}^{\mathrm{m} 2}, 37 / 2^{-}, 51.4 \mathrm{~m}\right)=7.33(9) \mu_{N} .
$$

Note that any hyperfine anomaly between the isotope ${ }^{175} \mathrm{Hf}$, on which the hyperfine field measurement was made, and ${ }^{177} \mathrm{Hf}^{\mathrm{m} 2}$ is not expected to exceed $0.1 \%$ and has been neglected.

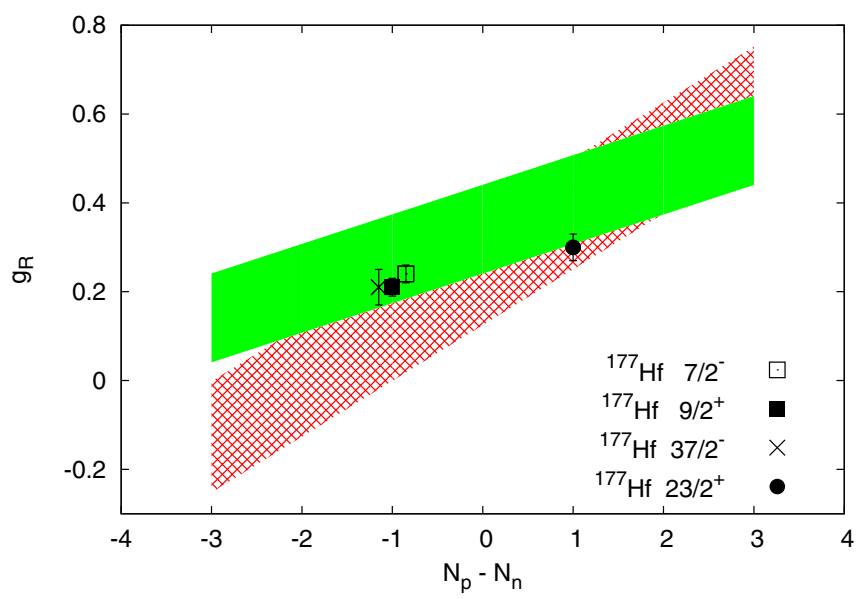

FIG. 9. (Color online) Plot of the collective $g_{R}$ as a function of $\Delta=N_{p}-N_{n}$. For ease of viewing the three data points at -1 have been slightly off-set along the $x$ axis. For discussion see text and Ref. [3]. 
TABLE III. Adopted $g_{K}$ value for individual quasiparticle states. Experimental data taken from [18].

\begin{tabular}{lcl}
\hline \hline Quasiparticle state & Adopted $g_{K}$ & \multicolumn{1}{c}{ Basis of adopted value } \\
\hline $\begin{array}{l}\text { Protons } \\
7 / 2^{+}[404]\end{array}$ & $0.765(25)$ & moments of $7 / 2^{+}$ground states in ${ }^{175,177,179,181} \mathrm{Ta}$ \\
$9 / 2^{-}[514]$ & $1.37(3)$ & moment of $9 / 2^{-} 6 \mathrm{keV}$ state in ${ }^{181} \mathrm{Ta}$ \\
Neutrons & & \\
$5 / 2^{-}[512]$ & $-0.48(2)$ & moment of $5 / 2^{-}$ground state of ${ }^{175} \mathrm{Hf}$ \\
$7 / 2^{-}[514]$ & $0.206(14)$ & moment of $7 / 2^{-}$ground state of ${ }^{177} \mathrm{Hf}$ \\
$9 / 2^{+}[624]$ & $-0.239(11)$ & moment of $9 / 2^{+}$ground state of ${ }^{179} \mathrm{Hf}$ \\
\hline \hline
\end{tabular}

\section{F. The magnetic moment of the 8 isomer in ${ }^{180} \mathrm{Hf}$}

Extraction of the magnetic moment from the observed resonance (Fig. 8) requires knowledge of the hyperfine field acting on Hf nuclei in the Ni lattice. Several previous values for this field have been reported, none of them of the necessary accuracy. The best would appear to be the Mössbauer study by Aggarwal et al. [17] who gave the value $B_{\mathrm{hf}}(\mathrm{HfNi})=$ $11.8(2.6) \mathrm{T}$. Using this value the observed ${ }^{180} \mathrm{Hf}^{\mathrm{m}}$ resonance yields the moment of the isomer to be $8.3(1.8) \mu_{N}$. Other, lower, reported $\mathrm{HfNi}$ fields give unrealistically high values for the nuclear moment. While the hyperfine fields at some neighboring elements have better measurements in nickel they do not allow an improved estimate for the field at the Hf nucleus. Clearly as it stands the present value is no improvement on the earlier results and the observed NMR result awaits an improved field value. This can be obtained, for example, by the observation of NMR in ${ }^{177} \mathrm{Hf}^{\mathrm{m} 2}$ activity in $\mathrm{Ni}$ where the frequency should be in the region of $35 \mathrm{MHz}$.

\section{DISCUSSION}

The variation in $g_{R}$ found in the analysis of this work (see below) prompted, in part, a comprehensive survey of nearly 100 high- $K$ bands in isotopes in this region [3]. The results have been recently published and draw two significant conclusions: that the assumption of additivity of quasiparticle contributions is a sound basis for estimation of the quasiparticle $g$ factor $g_{K}$ in multi-quasi-particle isomers in the majority of cases and that, based on analysis of the mixing ratio and branching ratio data, there is a wide and systematic variation of the collective $g$ factor $g_{R}$ which depends upon the quasiparticle make-up of the band head isomer. Underlying ideas concerning pairing and blocking in these isomers are discussed in the paper. In this section we review the extent to which the experimental results found in this work are consistent with the two conclusions described.

\section{A. Additivity of single quasiparticle $g_{K}$ factors to form $g_{K}$ for multi-quasi-particle states}

In Table III we give the $g_{K}$ factors adopted in the broad survey for those single-quasiparticle states which are relevant to the two isomers ${ }^{177} \mathrm{Hf}^{\mathrm{m} 2}$ and ${ }^{180} \mathrm{Hf}^{\mathrm{m}}$ for which the magnetic moments have been measured in this work. The $g_{R}$ factor makes a small contribution to the total magnetic moment since it is multiplied by the factor $I /(I+1)$ as compared to $I^{2} /(I+1)$ for $g_{K}$ [see Eq. (2)] and the spins of the two isomers are high, 37/2 and 8, respectively. Taking $g_{R}$ as $0.29(5)$ and assuming additivity, as described by Eq. (3) yields predicted moments of these isomers of 7.28(13) $\mu_{N}$ and 8.12(15) $\mu_{N}$ to be compared to the measured values, 7.33(9) $\mu_{N}$ and 8.3(18) $\mu_{N}$. Both results show agreement with the predictions based on additivity, consistent with the findings in [3].

\section{B. The variation of $g_{R}$}

Taking average values of the parameter $\left(g_{K}-g_{R} / Q_{0}\right)$ with $Q_{0}=7.2 \mathrm{eb}$ and $g_{K}$ assuming additivity in the multi-quasiparticle states, values of $g_{R}$ were extracted from this work for bands built on the $9 / 2^{+}, 23 / 2^{+}$, and $37 / 2^{-}$states in ${ }^{177} \mathrm{Hf}$. Results are given in Table IV which also includes $g_{R}$ for the band built on the $7 / 2^{-}$ground state of ${ }^{177} \mathrm{Hf}$. We reproduce in Fig. 9 the evidence of variation of $g_{R}$ as a function of the difference, $N_{p}-N_{n}$, of the numbers of unpaired quasiprotons and quasineutrons in the isomer reported in Ref. [3], showing the broad band ranges which, it is suggested, are caused by variation in the contributions from breaking specific quasiparticle pairs and the individual results from Table IV.

\section{CONCLUSIONS}

This paper reports new measurements of the magnetic properties of the isotopes ${ }^{177} \mathrm{Hf}$ and ${ }^{180} \mathrm{Hf}$ and their high- $K$ isomers. The magnetic moments of the $37 / 2^{-}$isomer in

TABLE IV. $g_{R}$ values extracted from band-head moments and band spectroscopic data. $Q_{0}$ taken as 7.2 eb throughout analysis.

\begin{tabular}{|c|c|c|c|c|c|c|c|c|}
\hline${ }^{177} \mathrm{Hf}$ & $37 / 2^{-}$ & 2740 & 2 & 3 & -1 & this work & [15] & $0.21(4)$ \\
\hline${ }^{177} \mathrm{Hf}$ & $9 / 2^{+}$ & 321 & 0 & 1 & -1 & [21] & this work & $0.21(2)$ \\
\hline${ }^{177} \mathrm{Hf}$ & $23 / 2^{+}$ & 1316 & 2 & 1 & +1 & additivity & this work & $0.30(3)$ \\
\hline
\end{tabular}


${ }^{177} \mathrm{Hf}$ and the $8^{-}$isomer in ${ }^{180} \mathrm{Hf}$ provide valuable additional evidence for the validity of additivity in estimating the quasiparticle $g$ factor $g_{K}$ in these deformed nuclei. Values of the collective $g$ factor, $g_{R}$, obtained from detailed $\gamma$ transition anisotropy measurements, are shown to be fully consistent with the recently revealed systematic dependence of this parameter upon the quasiparticle make-up of the bands involved.

[1] G. D. Dracoulis, F. G. Kondev, and P. M. Walker, Phys. Lett. B 419, 7 (1998).

[2] X. Wu, Z. H. Zhang, J. Y. Zeng, and Y. A. Lei, Phys. Rev. C 83, 034323 (2011).

[3] N. J. Stone, J. R. Stone, P. M. Walker, and C. R. Bingham, Phys. Lett. B 726, 675 (2013).

[4] A. Bohr and B. Mottelson, Nuclear Structure (W. A. Benjamin, Inc., Reading, MA, 1975).

[5] Low Temperature Nuclear Orientation, edited by N. J. Stone and H. Postma (North Holland, Amsterdam, 1986).

[6] U. Köster et al., Eur. Phys. J. Spec. Top. 150, 293 (2007).

[7] R. Eder (NICOLE Collaboration), Hyp. Int. 59, 83 (1990).

[8] T. L. Shaw and N. J. Stone, At. Data Nucl. Data Tables 42, 339 (1989).

[9] S. Muto, T. Ohtsubo, S. Ohya, and K. Nishimura, Hyperfine Interactions 158, 195 (2004).

[10] A. Nieminen et al., Phys. Rev. Lett. 88, 094801 (2002).

[11] J. R. Stone et al., Phys. Rev. C 76, 025502 (2007).

[12] H. J. Körner, F. E. Wagner, and B. D. Dunlap, Phys. Rev. Lett. 27, 1593 (1971).

\section{ACKNOWLEDGMENTS}

We thank Ajay Deo for helpful assistance with the experiment. The research was supported by the U.S. DOE Office of Science, the Ministry of Education and Science of Serbia (Project No. 171002), the European Commission via the ENSAR project, SNRS and IN2P3 (France), JSPS KAKENHI Grant No. 23540337 (Japan), and STFC (UK).

[13] K. S. Krane, S. S. Rosenblum, and W. A. Steyert, Phys. Rev. C 14, 650 (1976).

[14] K. S. Krane, in Ref. [6], Chap. 2.

[15] S. M. Mullins, A. P. Byrne, G. D. Dracoulis, T. R. McGoram, and W. A. Seale, Phys. Rev. C 58, 831 (1998).

[16] K. S. Krane, C. E. Olsen, and W. A. Steyert, Phys. Rev. C 10, 825 (1974).

[17] N. Aggarwal, B. Singh, A. K. Bhati, R. Bala, S. C. Bedi, and H. S. Hans, Hyperfine Interactions 13, 271 (1983).

[18] N. J. Stone, Table of Nuclear Magnetic Dipole and Electric Quadruple Moments, IAEA Nuclear Data Section Report No. INDC(NDS)-0594, April 2011.

[19] Y. Y. Chu, P. E. Haustein, and T. E. Ward, Phys. Rev. C 6, 2259 (1972).

[20] S. Büttgenbach, M. Herschel, G. Meisel, E. Schrödl, and W. Witte, Phys. Lett. B 43, 479 (1973).

[21] H. Hübel, C. Günther, K. Krien, H. Toschinski, K.-H. Speidel, B. Klemme, G. Kumbartzki, L. Gidefeldt, and E. Bodenstedt, Nucl. Phys. A 127, 609 (1969). 